\title{
Cancellation of plastic surgery operations on the day of surgery and its financial implications: an observational study
}

T

R. EMAMDEE 1 , H. Sivakumar ${ }^{2}$, H. Kuttambakam ${ }^{3}$, S. Pal ${ }^{4}$

${ }^{1}$ Consultant Anaesthetist, Mid Essex Hospitals NHS Trust - Chelmsford (United Kingdom), ${ }^{2}$ Staff Anaesthetist, Mid Essex Hospitals NHS Trust -

Chelmsford (United Kingdom), ${ }^{3}$ Consultant Anaesthetist, Southend University Hospitals NHS Trust - Southend on Sea (United

Kingdom), ${ }^{4}$ Professor, Anaesthesia, Mid Essex Hospitals NHS Trust - Chelmsford (United Kingdom)

\section{Background}

- The financial and time costs of same-day cancellation of plastic surgery procedures are considered substantial.

- There is a loss of 350,000 hr annually costing 400 million GBP in 153 acute trusts in England

\section{Objectives}

- To determine a numerical figure for the number of same day cancellations of elective plastic surgery patients in a specific time period

- To explore reasons for the cancellations

\section{Materials and Methods}

We reviewed elective lists in plastic surgery theatres at the end of each weekday and noted same day cancellations. The study period was 4 months (Apr 2017 to July 2017), Monday to Friday. We reviewed the notes in the ward and collected data including the reasons for the cancellation and whether post cancellation protocols as recommended by AAGBI were followed. We used this data to calculate theatre time loss in total and its implications

\section{Results}

- Total number of cancellations -50

- Reasons for cancellations

$$
\begin{aligned}
& \text {-Anaesthetic reasons - Uncontrolled DM - } 1 \\
& \begin{array}{ll}
\text { - Hypokalemia } & -1 \\
- \text { RTI } & -3
\end{array} \\
& \text { - No anaesthetist }-1 \\
& \text {-Surgical reasons - Surgery unnecessary - } 3 \\
& \text { - Low hemoglobin } \quad-1 \\
& \text { - Another patient turning up - } 1 \\
& \text { - Informed not to have surgery - } 1 \\
& \begin{array}{ll}
\text { - Patient factors } & \text {-Patient unwell - } 2 \\
& \text {-DNA - } 12(24 \%) \\
\text { - Theatre factors } & \text {-Delayed start -1 } \\
& \text {-Overrunning of theatre -7 (14\%) } \\
& \text {-Staff issues - } 13(26 \%)
\end{array} \\
& \text { - Others -Administrative error }-1 \\
& \text { - 'wannacry' ransomware-2 } \\
& \text { - Lack of bed - } 8 \text { (had other reasons too) }
\end{aligned}
$$

- Total theatre time lost 4636 mins ( approx. 77 hrs)

- $3 \%$ of elective operating time lost

- Cost - Theatre cost $-38,600 \mathrm{GBP}$ in total

$$
\begin{aligned}
& \text { - } 757 \text { GBP per case / } 500 \text { GBP per hour } \\
& \text { - 115,800 GBP per year } \\
& \text { - Other costs - Fine to trust }
\end{aligned}
$$

- Expenses claimed by patient

-Transportation costs

- Re-assessment / pathology costs

\section{Recommendations}

- To develop a robust protocol to deal with same day cancellations

- To improve recruitment and retention of staff

- To look at other novel measure to improve temporal and financial efficiency

\section{References}

\section{Limitations}

- It was a small time period and a summertime study. It may not be able to extrapolate results to winter period.

- Only plastics surgery cases were included and other specialities were not considered.

- It was also difficult to establish answers for some questions on the proforma (post-cancellation questions)

- Due to these factors, the real picture is possibly worse .

\section{Implications}

- Patient morale low and longer waiting times

- Physical difficulties to patient - fasting/transport/ childcare etc.

- Staff under utilisation and reduced operation theatre efficiency

- Financial implications for trust and strain on system to find the next available slot for the patients

- Other patients might be indirectly affected 